Original Research Paper

\title{
Influence of Degrading Calcium Phosphate on the Remodelling and Mineralisation of Avascular Osseous Tissue in a Rat Calvaria Model
}

\author{
${ }^{1,2}$ Tulio Fernandez, ${ }^{2}$ Carlos H. Valencia, ${ }^{1}$ Yuann Li, ${ }^{1}$ George A. Thouas, ${ }^{3}$ Donald F. Newgreen and ${ }^{1}$ Qizhi Chen \\ ${ }^{1}$ Department of Materials Engineering, Monash Medical School, Monash University, Clayton 3800, Australia \\ ${ }^{2}$ School of Dentistry, University of Valle, Cali, Colombia \\ ${ }^{3}$ Murdoch Childrens Research Institute, the Royal Children's Hospital, Flemington Road, Parkville, Victoria 3052, Australia
}

Article history

Received: 06-12-2014

Revised: $10-12-2014$

Accepted: 24-12-2014

Corresponding Author:

Qizhi Chen

Department of Materials

Engineering, Monash Medical

School, Monash University,

Clayton 3800, Australia

Tel: 61399053599 ;

Fax: 61399054940

E-mail: qzchen202@gmail.com

\begin{abstract}
Artificial bone graftingis the subject of intensive investigation for craniofacial reconstruction. Among the syntheticcandi date materials, those incorporating Calcium Phosphates $(\mathrm{CaP})$ have gained popularity, due to their osteoinductive properties. Despite many quantitative studies on the degradation rates of $\mathrm{CaP}$ biomaterials in vitro, there is lack of quantitative studies in relation to bone remodelling kinetics in vivo. In this investigation, we tested implants of degradable $\mathrm{CaP} /$ chitosan-based biomaterial in a rat calvaria model of critical-sized defects. We observed remarkable acceleration of bone growth in the initial stages $(\sim 0.8 \mathrm{~g} /$ day $)$, which was markedly higher than normal bone growth rates $(\sim 0.1 \mathrm{~g} /$ day $)$ in implant-free controls. The implanted CaP-based biomaterial significantly enhanced bone formation, with woven bone seen as early as 20 days and lamellar bone at 40 and 60 days post-implantation. However the mineralisation of cortical bone was delayed, due to the overly rapid degradation of the biomaterial: This therapeutically important issue has never been identified before to our best knowledge. A theoretical analysis revealed that during degradation $\mathrm{CaP}$ can be rapidly released from the new bone matrix. Hence, while the degradable $\mathrm{CaP}$ biomaterial was found to be highly osteoconductive in vivo, future modifications would seek to optimize degradation efficiency for more sustained release of mineral ions, to enhance bone mineralisation at later stages of the healing process after implantation.
\end{abstract}

Keywords: Dental Graft, Calcium Phosphate, Rat Calviria, Bone Growth Rate, Mineralisation

\section{Introduction}

The loss of teeth and supportive tissues in the jawbone often causes severe bone resorption, which hinders the healing process for dental implants, as well as recovery of physiological function (e.g., chewing, speech) and cosmetic appearance (Vignoletti et al., 2012). Current approaches in bone grafting for dental implants are dominated by autologous grafts and by allografts; however biological bone grafts have several shortcomings. These include donor tissue shortage and morbidity in autologous grafting and immune rejection or transmission of pathogens (e.g., HIV) in allografting (Burg et al., 2000). Over the past decade, artificial bone grafts have been investigated using various synthetic materials, including Hydroxyapatite (HA), Calcium Phosphates (CaP), polyesters, chitosan and their composites (Chen et al., 2012). Among these biomaterials, those containing $\mathrm{CaP}$ have been extensively tested (Bohner et al., 2012; Chai et al., 2012) because of their osteoinductive properties (Geffre et al., 2010; Thormann et al., 2013).

Osteoinduction refers to the heterotopic or ectopic formation of new bone at a wound site, due to the biological actions of growth factors (e.g., Bone Morphogenetic Proteins (BMPs) and bone minerals (e.g., $\mathrm{CaP}, \mathrm{Sr}, \mathrm{F}$ ) (Habibovic and de Groot, 2007; Barradas et al., 2012). In addition, specific physico-chemical properties of the biomaterial, such as particle size (Ignjatovic et al., 2010), surface area, crystallinity, porosity and composition are also able influence bone formation (Lu et al., 2002; Lange et al., 2011). It has been reported that particle sizes ranging from 80 to $300 \mu \mathrm{m}$ in diameter 
can induce ectopic bone formation, whereas particles greater $500 \mu \mathrm{m}$ are not osteoinductive (Balaguer et al., 2010). In vivo studies also indicate that a specific surface area above a threshold level of $1.0 \mathrm{~m}^{2} / \mathrm{g}$ is critical for $\mathrm{CaP}$ to induce osteogenesis, suggesting the importance of optimizing surface areas of bone scaffolds (Chai et al., 2012). It is likely that the influences of particle size and specific surface area on bone formation are mediated via their effects on the degradation rate of $\mathrm{CaP}$ materials.

Biphasic materials containing mixtures of HA with $\mathrm{CaP}$ demonstrate simultaneously increased matrix solubility and osteoinductivity (Yuan et al., 2006), whereas pure HA is too stable and amorphous TriCalcium Phosphate (TCP) is too easily solubilised to be appreciably osteoinductive (Arinzeh et al., 2005). Based on this, we envisage that the osteoinductivity of CaPbased biomaterials is directly controlled by their degradation rates. Other materials properties (e.g., particle size, surface area, crystallinity, porosity and composition) influence osteoinductivity indirectly via degradation rates. Hence, degradation kinetics of biomaterials is acommon and important factor that can affect new bone formation.

Despite many quantitative studies on the degradation rates of $\mathrm{CaP}$ biomaterials in vitro (Bohner et al., 2012; Chai et al., 2012; Barradas et al., 2012), there is lack of similar studies in relation to bone remodelling kinetics in vivo. Therefore, a primary objective of this study was to quantitatively investigate the influence of degrading CaPbased biomaterials on the remodelling of a vascular osseous tissue. To this end, we used the rat calvaria model because of its lack of intra-bone vascular networks (Pannarale et al., 1997). Given that dentinis an avascular tissue, this model particularly suits the evaluation of dental biomaterial in vivo.

\section{Materials and Methods}

\section{Biomaterial Preparation}

The biomaterial used in this study was a paste made from solid bioceramic powder and anaqueous chitosan solution ( $2 \mathrm{wt} \%, \mathrm{pH}=4.5)$, which was purchased from Polimar Cienciae Nutricao S.A. Chitosan is another biomaterial being intensively investigated for its ability to enhance osteoinductivity of CaP-based materials (Kim et al., 2008). For these reasons, the biomaterial used in this study is a composite of $\beta$-Tricalcium Phosphate ( $\beta$-TCP) and chitosan in its acetylated form.

The bioceramicpowder was amixture of $\beta$-TCP $\left(\right.$ Emprove $\left.^{\circledR}\right)$, calcium oxide and zinc oxide, all purchased from Merck ${ }^{\circledR}$. The particle size of each powder was measured by laser granulometry using a Mastersizer 200 (Malvern Instruments). The analysis was conducted using a laser diffraction liquid method on the following suspensions: $\beta$-TCP dispersed in propanol and $\mathrm{CaO}$ and $\mathrm{ZnO}$ dispersed in water. The particle diameters of the $\beta$-TCP, $\mathrm{CaO}$ and $\mathrm{ZnO}$ powders were 15, 3 and 9 $\mu \mathrm{m}$, respectively. $\mathrm{CaO}$ was incorporated mainly for adjustment of $\mathrm{pH}$ of the composite and the minor addition of $\mathrm{ZnO}$ aimed at bone regeneration at early stages, as indicated by increased markers of osteoblast differentiation, matrix maturation and bone mineralisation in a previous work (Fielding and Bose, 2013).

A series of composites (100 samples) was systematically prepared from bioceramic powders of different $\beta$-TCP: $\mathrm{CaO}$ : $\mathrm{ZnO}$ ratios and mixed with the liquid chitosan solution at various solid/(solid+liquid) percentages. The $\mathrm{pH}$ values of these composites were measured. The composites ( 20 samples) with a $\mathrm{pH}$ value between 6.5 and 8.5 were considered provisionally to be safe for biological environments and were evaluated in vitro and the rest (80 samples) were discarded because of anticipated toxicity. The best formula in terms of cytocompatibility (section 3.1 ) in vitro is listed in Table 1, which had a $\mathrm{pH}$ value of $\sim 7.5$ and thus was subsequently chosen for the rat wound correction study.

The composite mixture was prepared as follows. The three ceramic powders were gently mechanically mixed for $\sim 5 \mathrm{~min}$ and then the mixture was dried in a microwave oven for $20 \mathrm{~min}$. Each of these dried mixtures was added to the chitosan solution according to the designed percentage (Table 1) to produce a paste. The compressive strength of the paste was $\sim 0.5 \mathrm{MPa}$. The crystallinity of $\beta$-TCP was approximately $30 \%$, as provided by the supplier. The $\mathrm{CaO}$ and $\mathrm{ZnO}$ powders were amorphous.

\section{Evaluation of Cytocompatibility in Vitro}

The biocompatibility of materials in vitro were tested by exposure of proliferating cells to the extract media conditioned by material samples as they degrade, according to the standard cytotoxicity assessment study set by the International Standardization Organization (ISO 10993). In this method, extracts were obtained by soaking the test and control materials in separate cell culture media (DMEM supplemented with 10\% Fetal Calf Serum (FCS), 1\% L-glutamine and $0.5 \%$ penicillin/streptomycin) under conditions of $0.2 \mathrm{~g} \mathrm{~mL}^{\square 1}$ of culture medium for $24 \mathrm{~h}$ at $37^{\circ} \mathrm{C} / 5 \% \mathrm{CO}_{2}$ in an incubator. HA was used as a positive control and tissue culture medium (DMEM supplemented with 10\% Foetal Calf Serum (FCS), 1\% L-glutamine and $0.5 \%$ penicillin/streptomycin) alone was used as the negative control. All material samples were sterilized using 70\% alcohol/de-ionized water solution. Osteoblast-like MG63 cells from the American Type Culture Collection (ATCC) were seeded in culture media at a density of approximately 2000 cells/well in 96-well tissue culture plates (BD Falcon ${ }^{\mathrm{TM}}$ ). When cells reached $70 \%$ confluence (in approximately 2 days), the medium in each well was entirely replaced with $0.2 \mathrm{~mL}$ of extract media. All cultures were then further incubated for two days. 
Table 1. Formula of biomaterials used in the work

\begin{tabular}{|c|c|c|c|}
\hline Bioceramic powder (wt. \%) & & & Composite (wt. \%) \\
\hline$\beta$-ТCP & $\mathrm{CaO}$ & $\mathrm{ZnO}$ & Bioceramic powder/composite \\
\hline 96 & 2.5 & 1.5 & 40 \\
\hline
\end{tabular}

At the end of the incubation period, cytotoxicity was quantified using a commercial kit, Tox-7 (Sigma-Aldrich). Spent culture media were collected and the degree of cell death was determined by measurement of Lactate Dehydrogenase (LDH) levels in the culture media ('DEAD LDH'), as described previously (Liang et al., 2010). Each well containing living cells was then filled with $0.2 \mathrm{~mL}$ fresh cell culture medium and cells were treated with Tox-7 lysis solution. These lysates were then used to determine the cellular LDH content, which equates to the number of living cells per well ('LIVE LDH'). LDH level was determined by measuring the absorbance of the supernatant from the centrifuged medium at $490 \mathrm{~nm}$ (after subtraction for background absorbance at $690 \mathrm{~nm}$ ) using a multi well plate format UV-vis spectrophotometer (Thermo Scientific). The absorbance results of $\mathrm{LDH}$ can be converted to the number of cells according to a linear standard curve. Hence, cytotoxicity can be expressed as follows Equation 1:

Percentage of dead cells (\%)

$=\frac{\text { DEAD LDH }}{\text { DEAD LDH }+ \text { LIVE LDH }} \times 100$

\section{Animal Model}

Various animal models of bone repair with the anatomical capacity to recreate a Critical-Sized Defect (CSD) have been developed for biomaterials research (Kochi et al., 2009; Gosain et al., 2000), but the 5-mm rat calvarial bone defect is one of the most frequently used for in vivo studies (Mills, L.A. and A.H. Simpson, 2012; Develioglu et al., 2009; Jones et al., 2007). Very recently, a systematic review on calvarial CSD correction (Vajgel et al., 2013) indicated that only 1.6\% of such $5.0-\mathrm{mm}$ defects heal completely with newly formed bone. In this study, 12 male Wistar rats, which were 4-months old and weighed $300 \mathrm{~g}$ on average, were used. The rats were randomly divided into three groups of 4 rats, to be examined respectively at 20-, 40- and 60day time points. Two bone defects $(5 \mathrm{~mm}$ in diameter and full depth of $\sim 0.8 \mathrm{~mm}$ ) were made in each rat calvaria (control and test) (Fig. 1a). The experimental protocols and the animal care was approved and supervised by the Animal Ethics Committee of the University of Valle (Cali, Colombia).

\section{Surgical Procedure}

Operations were performed on the rats using general anaesthesia, i.e., ketamine $\left(50 \mathrm{mg} \mathrm{mL}{ }^{-1} ; 0.7 \mathrm{mg} \mathrm{kg}^{-1}\right)$, xilacin $\left(2 \%, 0.6 \mathrm{mg} \mathrm{kg}^{-1}\right)$ and acepromazine maleate $(10$ $\mathrm{mg} \mathrm{mL} \mathrm{m}^{-1}, 0.6 \mathrm{mg} \mathrm{kg}^{-1}$ ). Two circular bone defects were introduced using a trephine bur with a dental implant surgical handpiece (400 rpm). After washing the rat skull with a physiological saline solution, the right side skull bone defect was filled with chitosan/ceramic paste and the left one was left empty as a control (Fig. 1b). The scalp wounds were closed by suture. The rats were sacrificed at 20,40 and 60 days post-implantation. To minimise experimental variability, the same surgeon performed all procedures.

\section{Histology and Electron Microscopy Sample Preparation}

After animals were euthanized, the experimental bone defects were retrieved and fixed in $2.5 \%$ glutaraldehyde Tris Buffered Saline (TBS) (0.01M, pH 7.4) solution for $48 \mathrm{~h}$ at $4^{\circ} \mathrm{C}$. After washing with TBS (0.01M, pH 7.4), control samples and two samples of each experimental group were decalcified in $15 \%$ Ethylenediaminetetraacetic Acid (EDTA) water solution $(\mathrm{pH} 7.3)$ at $4^{\circ} \mathrm{C}$ for 2 months and the other two samples of each test group remained undecalcified. After rinsing in TBS $(0.01 \mathrm{M}, \mathrm{pH} 7.4)$, the bulk decalcified and undecalcified samples were dehydrated in increasing ethanol concentrations (70-100\%) and embedded in paraffin wax for optical microscope histology or Poly (Methyl Methacrylate) (PMMA) for transmission electron microscopy (Bateman et al., 2012). Then, 5$\mu \mathrm{m}$ thick slides for histology or 50-70 nm TEM were sectioned along the coronal plane (Fig. 1b) in cooling water with a microtome or an ultrathin microtome, respectively. Histological slides of decalcified bone were stained with Haematoxylin-Eosin (H\&E), whereas those of undecalcified bone were stained with Goldner's (Masseon) trichrome.

\section{Histomorphometric Analysis}

The above stained samples were imaged with an Aperio Scan Cope ${ }^{\circledR}$ Turbo scanner (Aperio Technologies, Vista, CA, USA/Serial Number AT1681). All scans were conducted at the same resolution and magnification (i.e., 0.497 microns per pixel, $20 \times$ ). The images of the region of interest were then processed with Adobe Photoshop ${ }^{\circledR}$ version CS2 (9.0) to obtain the masks. In this process, the features of interest were designated in black and the rest of the examined area was designated in white and thus, a black-and-white image (i.e., mask) was created. Image $J^{\circledR} 1.46 \mathrm{r}$ software was used to measure the area percentage (Ar. $\%$ ) of the selected features in the examination field (Egan et al., 2012). 


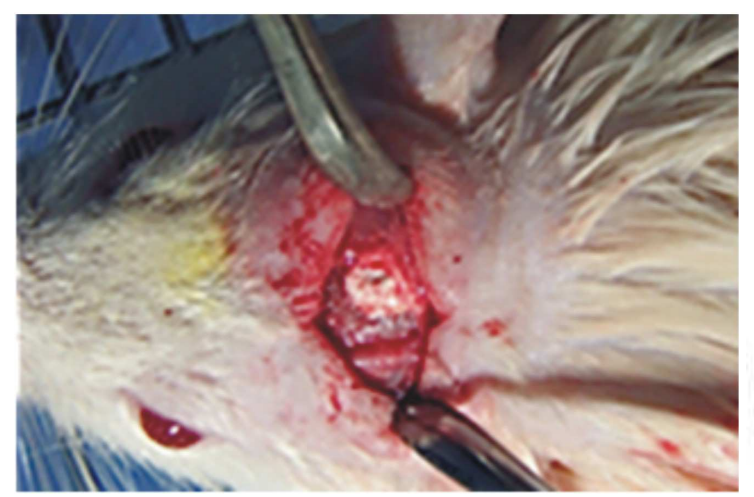

(a)

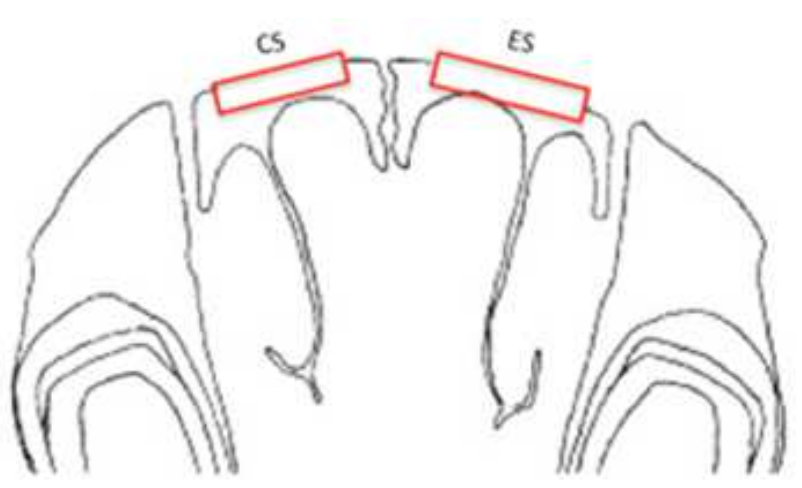

(b)

Fig. 1. (a) Surgical sites indicated on the exposed rat calvaria and (b) diagram of the coronal view of Experimental Site (ES-left) and Control Site (CS-right) which, with surrounding host bone, were retrieved at the end of the experimental period. The histological samples were sectioned across the middle region of each sample along the coronal plane

\section{TEM Observation}

The TEM foils were examined with a Tecnai 20 microscope, with soft tissue regions examined at $100 \mathrm{kV}$ and bone tissue regions at $200 \mathrm{kV}$.

\section{Statistical Analysis}

In vitro experiments were performed with six samples per experimental group and in vivo experiments were performed with 4 animals per experimental group. The statistical outputs are shown in the form of a mean with Standard Error $( \pm \mathrm{SE})$. A one-way analysis of variance (Mikhailenko et al., 2013) with Tukey's post hoc test was performed to analyse the significant differences and the significance levels were set at a $p$ value of less than 0.05 .

\section{Results}

\section{In vitro Evaluation of Cytocompatibility}

Cellular toxicity was visible in the cultures containing extracts of CaP-chitosan pastes of $\mathrm{pH} 6.8$ and 7.2 (Fig. 2c and d), while the media containing the extracts of the pastes of $\mathrm{pH} 7.5$ and 7.8 were found to support proliferation of MG63 cells (Fig. 2e and f). Quantitative LDH measurements confirmed that the cytocompatibility of the composite paste of $\mathrm{pH} 7.5$ was similar to both culture media and HA controls, with no significant differences detected in cell death percentage between the optimised paste and control groups (Fig. 3). Hence, the subsequent in vivo studied were conducted using the paste of $\mathrm{pH}$ 7.5.

\section{Histological Analysis}

All surgical procedures were performed without complications. Histological examination (Goldner's Messon trichrome) revealed that the control defects remained empty, with little new bone formed up to 40 days post-implantation and the amount of new bone was considerable only in the samples of the 60-day treatment group ( 20 Ar. \% of the defects) (Fig. 4). These results are in agreement with previous work on bilateral calvarial defects of $5 \mathrm{~mm}$ diameter, which reported that the area percentage of new bone formed in untreated control defects was approximately 20 and $23 \%$ at 2 and 3 months, respectively (Vajgel et al., 2013).

In contrast, the formation of new bone was more extensive in all the experimental sites implanted with the CaP-based biomaterial, with defects being filled with new soft and/or hard tissues (Fig. 5). In the 20day tissue samples (Fig. 5a), the central region of the defect was occupied by a mixture of biomaterial particles and soft fibrous tissue: This was defined as Region I. Newly formed bone was defined as Region II (Fig. 5a). By 40 days after implantation, the defects were predominately occupied by new bone tissue, with bone marrow scattered in some discrete areas in the intermediate zone of the defects (Fig. 5b). After 40 days, no significant change was observed histologically (Fig. 5c).

The area percentages of Region I, II and bone marrow are given in Fig. 6. Region I was about $50 \%$ by area at 20 days but nearly completely replaced by new bone by day 40 (Fig. 6). Assuming the bone was growing at a reasonably constant rate, the average growth rate of the bone is thus estimated to be 2.5 $\mathrm{mm} / 40$ days $=62.5 \mu \mathrm{m} \mathrm{day}{ }^{-1} \approx 1.0 \times 10^{-3} \mu \mathrm{m} / \mathrm{s}$ in thickness. This rate is remarkably higher than the growth rates of bone remodelling in rats of the same age (Fig. 7). It is estimated by extrapolation of the data in Fig. 7, which was retrieved from literature (Hansson et al., 1972), that the bone growth rate in rats of 4-months old could be as low as several $\mu \mathrm{m} / \mathrm{day}$, which has also been reported by another study (Raman, 1969).

An alternative way to quantify growth rate is weight per day. The density of bone is $\sim 2 \mathrm{~g} / \mathrm{cm}^{3}$. The weight of a 5 $\mathrm{mm}$-diameter, $0.8 \mathrm{~mm}$-thickness bone defect is $\sim 0.03 \mathrm{~g}$. 


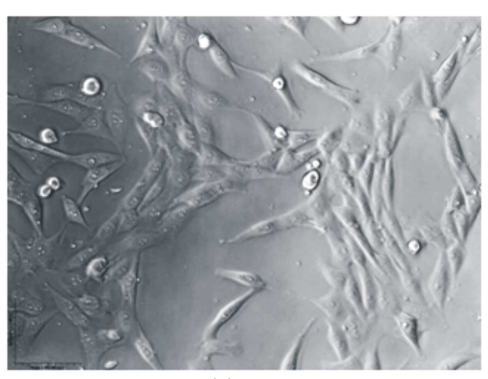

(a)

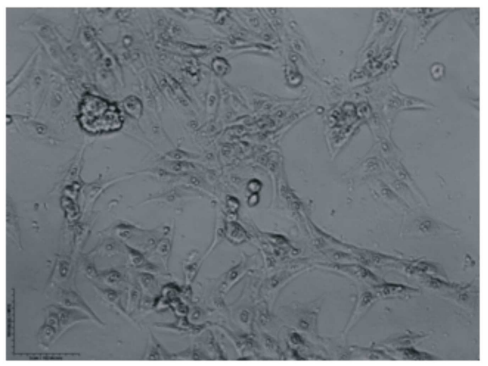

(c)

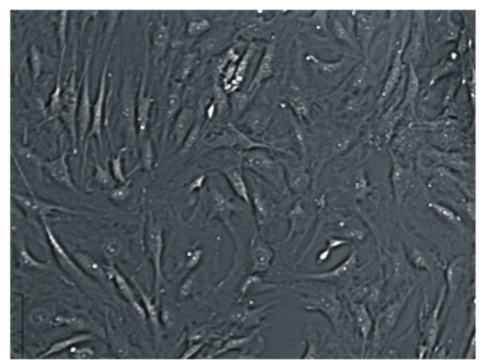

(e)

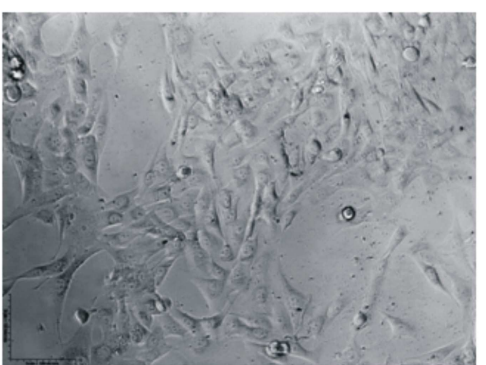

(b)

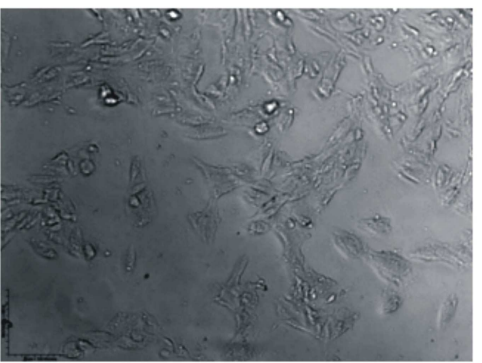

(d)

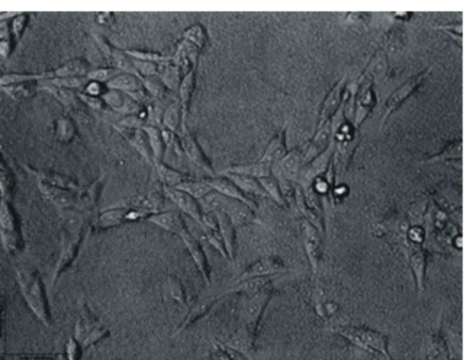

(f)

Fig. 2. Images of MG63 cells after one day culture in extractmedia, which were prepared by soaking test materials in tissue culture medium for $24 \mathrm{~h}$ (a) Negative control (culture medium only) and (b) positive control of hydroxyapatite samples CaP-chitosan composite of $\mathrm{pH}$ (c) $\mathrm{CaP}-\mathrm{Ch} \_\mathrm{pH}=6.8$, (d) $\mathrm{CaP}-\mathrm{Ch} \_\mathrm{pH}=7.2$, (e) $\mathrm{CaP}-\mathrm{Ch} \_\mathrm{pH}=7.5$ and (f) $\mathrm{CaP}-\mathrm{Ch} \_\mathrm{pH}=7.8$ Magnification is the same for the six images

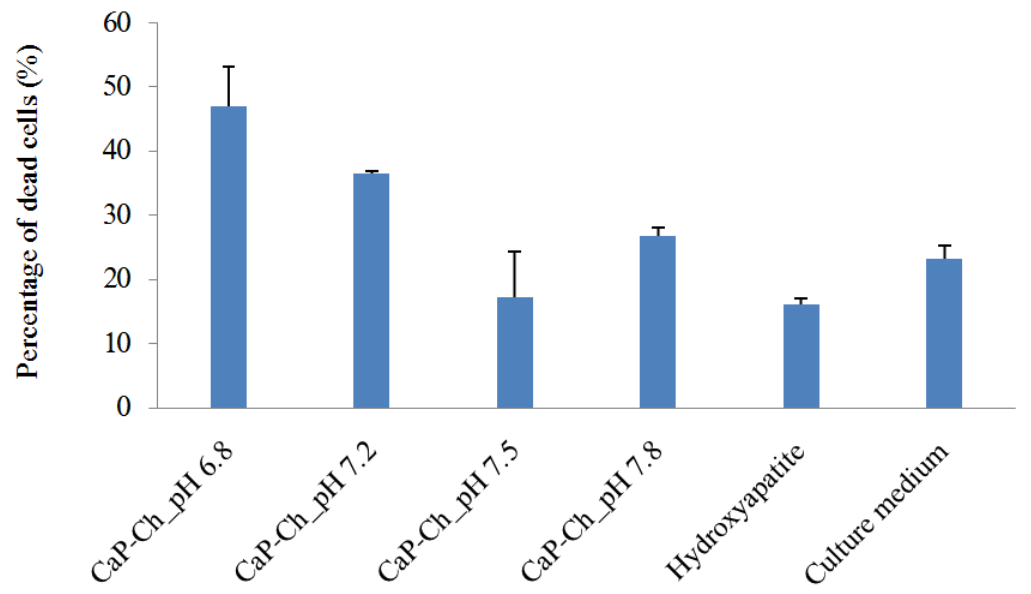

Fig. 3. Cytotoxicity of the CaP-chitosan composites determined by measuring the release of LDH after two days of culture. The positive and negative control group was HA and standard culture medium, respectively. The percentage of dead cells in CaPchitosan composite of $\mathrm{pH} 6.8$ and 7.2 were significantly elevated, but at $\mathrm{pH} 7.5$ was not significantly different from either the negative control or positive control groups $(p>0.05)$. The cell death percentage in the CaP-chitosan of $\mathrm{pH} 7.8 \mathrm{was}$ significantly different from that of the positive control, but not with that of the negative control 


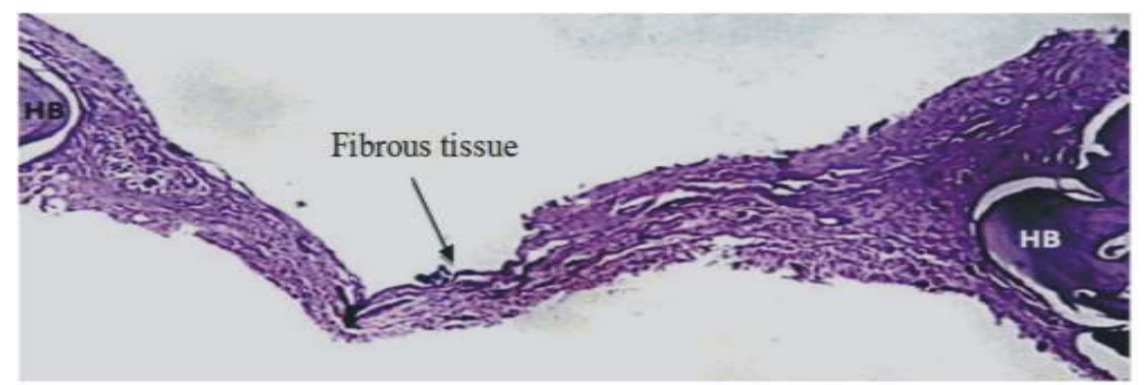

(a)

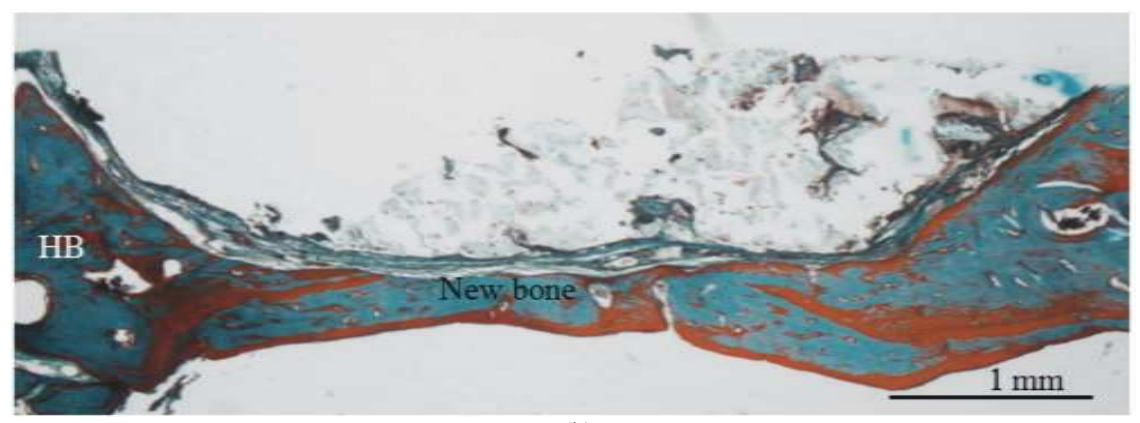

(b)

Fig. 4. Low power view of sections of the undecalcified defect tissue from rats that had no implantation of biomaterial particles taken (a) 40 (H\&E stained) and (b) 60 (Golders's Masson trichrome stained) days post-surgery. Almost no new bone formation was observed in the defects retrieved at day 20 and 40 post surgery. After 60 days, new bone was observed, occupying approximately $20 \mathrm{Ar}$. \% of the defect tissue. HB stands for host bone
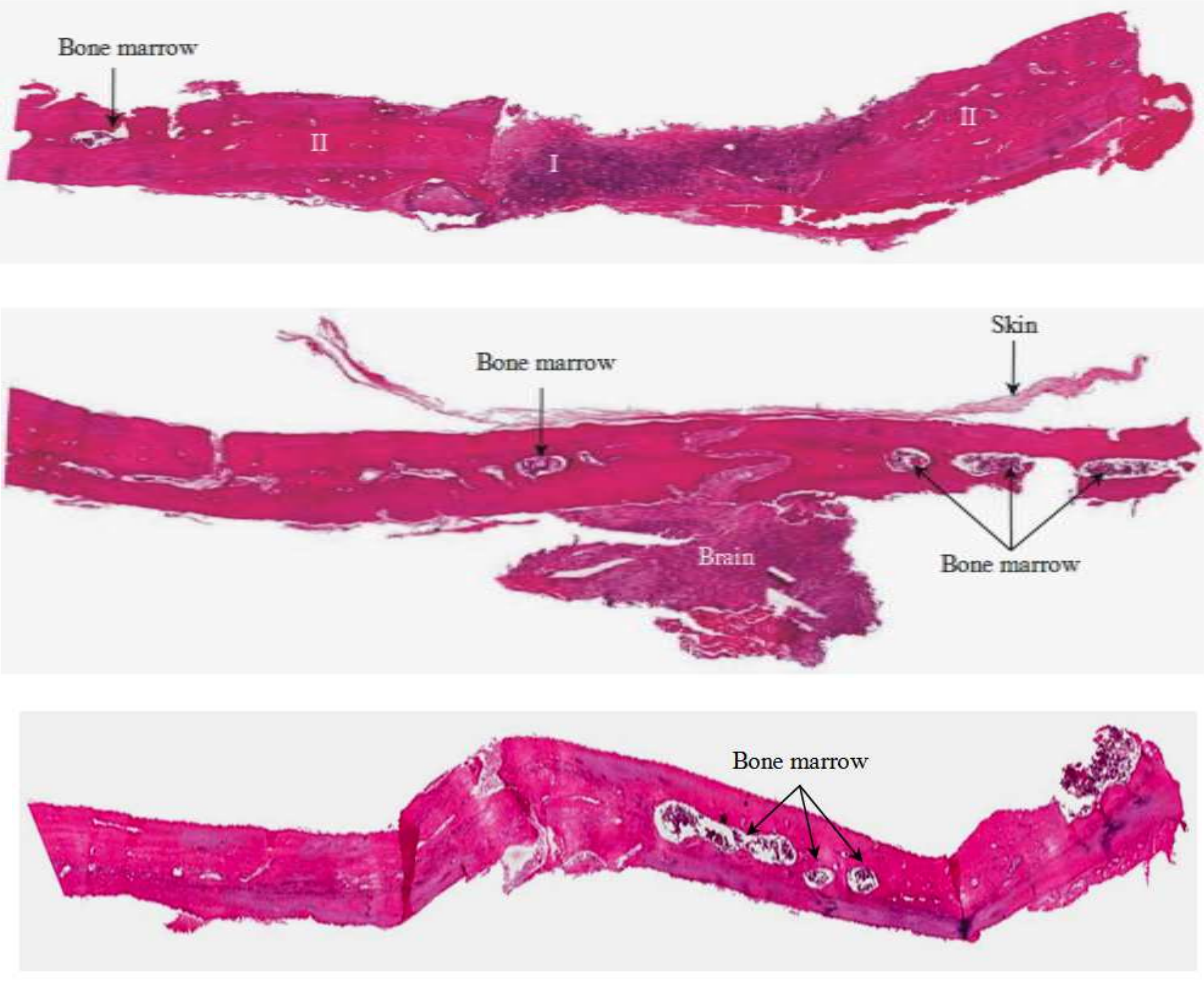

$1 \mathrm{~mm}$

Fig. 5. Low power images of H\&E stained sections of decalcified CaP biomaterial-implanted tissue (a) 20, (b) 40 and (c) 60 daysafter implantation Tissues in Region I consisted mainly of fibrous tissues and CaP particles. RegionII consisted of newly formed bone 


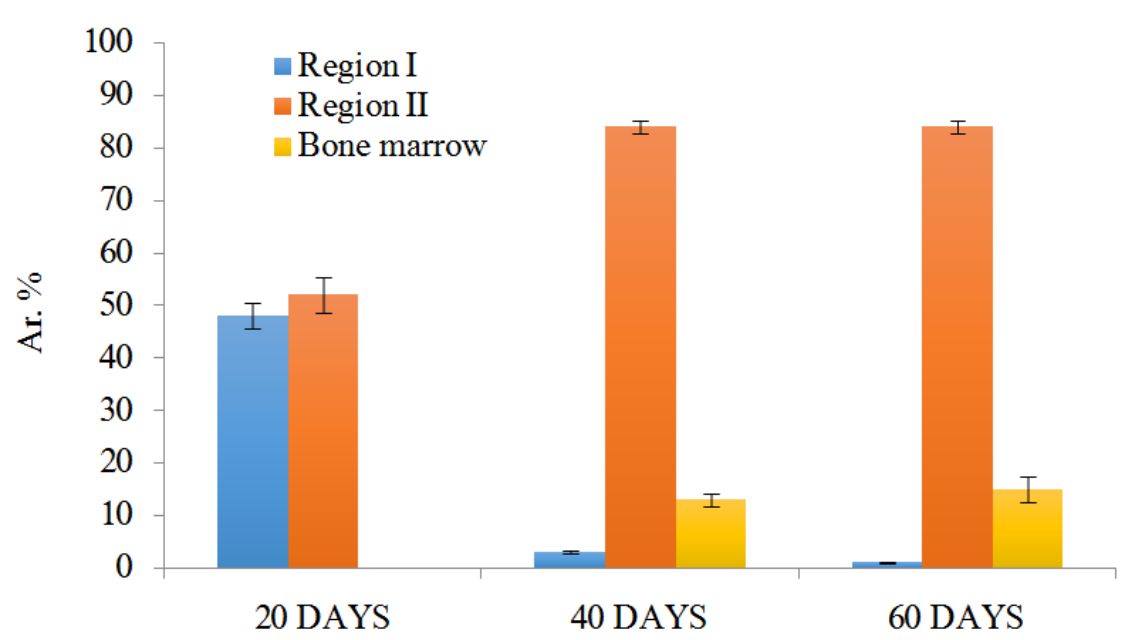

Fig. 6. Area percentages of Region I, II and bone marrow in samples of the experimental group retrieved at day 20,40 and 60 . The differences between 20 days data $(*)$ and 40 or $(60)$ days data $(* *)$ were significant $(p<0.01)$. There was no significant difference between 40 and 60 days data

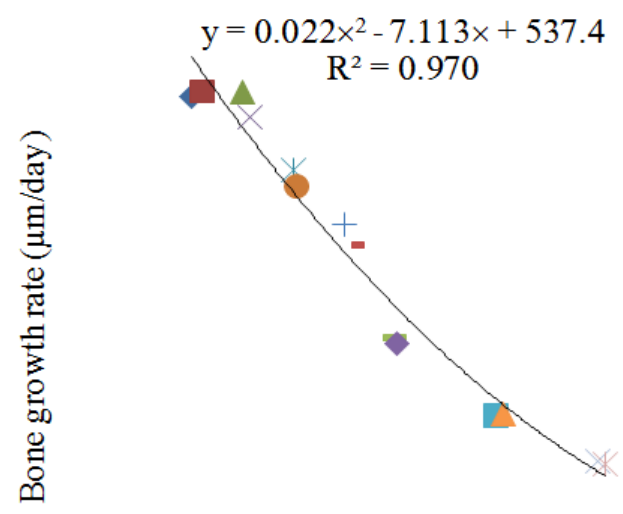

Age of rats (day)

Fig. 7. Bone (tibia) growth rate vs age of rats (male). Data were retrieved from (Bateman et al., 2012)

Assuming equivalent bone density, the bone growth rate of CaP-grafted defects is thus estimated to be $0.03 \mathrm{~g} / 40$ days $\approx 0.8 \mathrm{~g} /$ day and for control defects estimated as $0.03 \mathrm{~g} \times 20 \% / 60$ days $\approx 0.1 \mathrm{~g} /$ day. Again the growth rate of implanted defects was significantly higher than the normal remodelling in rats of the same age.

High power imaging of Region I revealed the disaggregated biomaterial particles embedded in the fibrous tissue at day 20 post-implantation (Fig. 8). Giant cells, as well as blood vessels, were observed surrounding biomaterial particles. Histomorphometric analysis indicated that the area percentages of biomaterial particles in region I were approximately $55 \pm 8 \%$.

The tissue of Region II was avascular cortical bone, characterised by osteocytes and canaliculi (Fig. 9). There were more osteocytes in the cortical bone of 40-days sample (Fig. 9a) than in the bone of 60-days group (Fig. 9b). Almost no biomaterial particles could be observed in Region II under optical microscopy. The newly formed bone was stained red by Goldner's trichrome after both 40 and 60 days post implantation. Goldner's trichrome stain is sensitive to the level of mineralisation in bone and thus has been used to discriminatenon-mineralised bone (immature bone, red) and mineralised bone (mature bone, green/blue) in undecalcified samples. The red stain by trichrome in 40 and 60 days samples indicated that the mineralisation in the bone remained poor after 60 days implantation, apparently not being enhanced by the degraded CaP-based material.

\section{TEM Examination}

TEM examination revealed that many micro- or sub-micron-sized particles presented in what were most likely giant cells in Region I of the 20 days samples (Fig. 10a) and these sub-micro-sized particles were broken down further into nano-sized particles (Fig. 10b).Very fine biomaterials particles were still visible in newly formed woven bone tissue in the 20 days samples (Fig. 10c). However, it was hard to find any biomaterial debris in lamellar bone in 40 and 60 days samples (Fig. 10d).

\section{Summary of Histological Observations}

The degradation of $\mathrm{CaP}$ particles occurred primarily in Region I. Large agglomerates were first infiltrated and fragmented by fibrous tissue (Fig. 5a) and sub-micron sized particles were phagocytosed by giant cells, further broken down to nano-sized particles, which were fused by lysosomes (Fig. 10b). The $\mathrm{CaP}$ material degradation was virtually complete and replaced entirely by new bone.

The critical sized defects were nearly completely filled with new bone tissue after 40 days postimplantation and healed completely with nonvascularised cortical bone. 


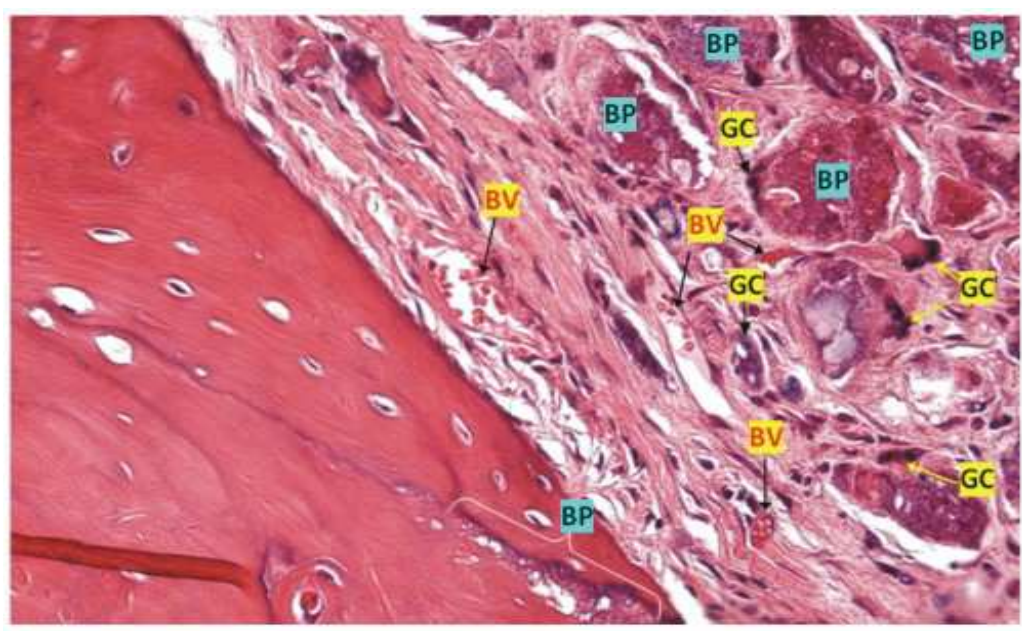

$50 \mu \mathrm{m}$

Fig. 8. Three representative high power views of H\&E stained tissue in Region I seen in samples of CaP-biomaterial implanted bone defects retrieved at 20 days post implantation. Biomaterial particles (BP) were surrounded by fibrous tissue with prominent Blood Vessels (BV), where Giant Cells (GC), presumed to be macrophage polykaryons consistent with a foreign body reaction, were noted in proximity to the $\mathrm{BP}$

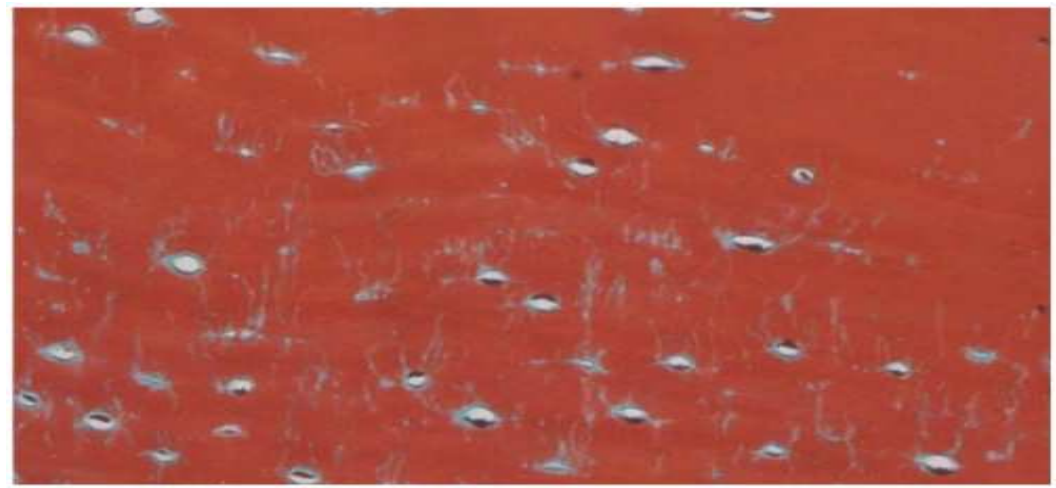

(a)

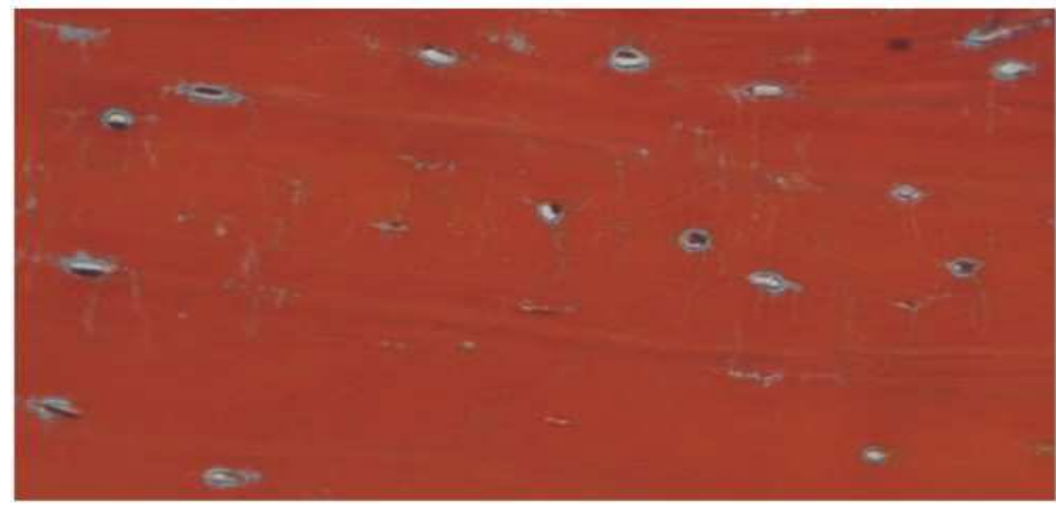

(b)

$50 \mu \mathrm{m}$

Fig. 9. High power of Goldner'strichrome stained avascular cortical bone after (a) 40 and (b) 60 days implantation

However, after 40 days implantation, the mineralisation of the non-vascularised cortical bone remained poor, this process apparently not being enhanced by the CaP-based biomaterial. 

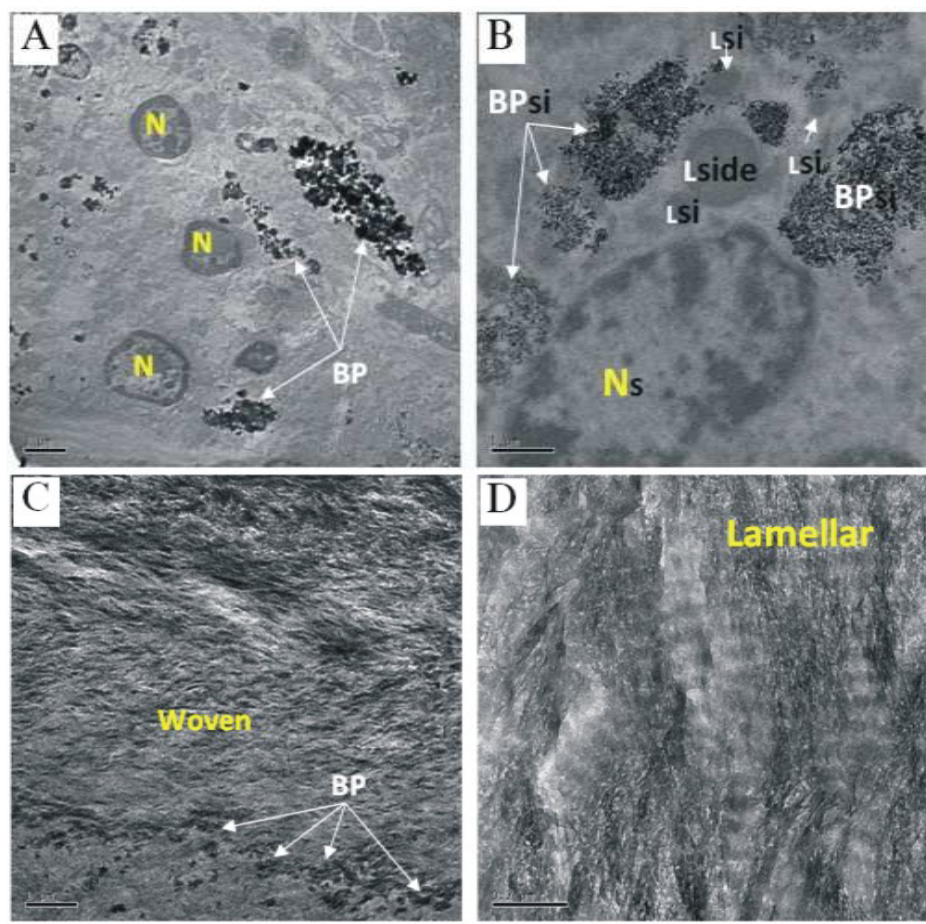

Fig. 10. Transmission Electron Microscope (TEM) images of (a) biomaterial particles inside a giant cell and (b) the particles near the nucleus of the cells, in Region Iin samples retrieved at 20 days post implantation. (c) Woven bone observed in a sample retrieved at 20 days post implantation. (d) Lamellar bone observed in a sample retrieved at day 40 post implantation; similar morphologies were also observed in samples retrieve at day 60 (not shown). BP indicates biomaterials particles. L indicates lysosome and $\mathrm{N}$ indicates a cell nucleus

\section{Discussion}

The degradation of the implanted CaP-based particles primarily occurred in Region I where the amount of biomaterial particles, as indicated by significant reduction in average Ar. \% of particles from initial 100\% at day zero, to $50 \%$ at 20 -days post implantation. The possible fates of sub-micronsized particles produced upon degradation of the material include phagocytosis by macrophages (Fig. 10a and b), retention in the newly formed bone (Fig. 8 and 10c) and dissolution (i.e., ionising into $\mathrm{Ca}^{2+}, \mathrm{Zn}^{2+}$ and $\left.\mathrm{PO}_{4}{ }^{3-}\right)$ during remodelling of the bone matrix. Part of the released $\mathrm{Ca}^{2+}, \mathrm{Zn}^{2+}$ and $\mathrm{PO}_{4}{ }^{3-}$ ions could participate in the calcification of the newly formed bone. However excessive amounts of minerals are likely to have been removed via cellular mechanisms, as indicated by the evidence of foreign body reaction (Fig 10b). It is likely that the observed bone marrow and osteocytes (as well as canaliculi) in the newly formed bone (i.e., Region II, Fig. 9) could serve as efficient channels in removing excess mineral ions.

The migration kinetics of excessive ions in bone can be predicted using one-dimensional Fick's second law:

$$
\frac{\partial C(x, t)}{\partial t}=\frac{\partial}{\partial x}\left(D \frac{\partial C(x, t)}{\partial x}\right)
$$

where, $C$ is the concentration of the diffusing ion, which is the function of time $t$ and distance $x . D$ is diffusion coefficient of the ion in the bone matrix, which is $10^{\square 7}$ $\mathrm{cm}^{2} / \mathrm{s}$ in bone (Fernandez-Seara et al., 2002). If the diffusion coefficient is independent of concentration, Equation 2 simplifies to:

$\frac{\partial C(x, t)}{\partial t}=D \frac{\partial^{2} C(x, t)}{\partial x^{2}} t=0, C=C_{0}=1$ at $0 \leq x \leq \infty$

The concentration $C$ of mineral ions is approximately considered as 1 at the biomaterial site and zero at the sink site (Fig. 11). When the ions migrate from degrading particles to blood vessel to reduce the concentration from $C=1$ to the physiological level (the weight percentage of bone minerals in bone is 0.7 ). The boundary conditions are summarised as follows Equation 4:

$t>0,\left\{\begin{array}{l}C=C_{s}=0 \text { at } x=0 \\ C=C_{0}=1 \text { at } x=\infty\end{array}\right.$

Application of these boundary conditions to Equation 3 yields the solution:

$\frac{C(x, t)-C_{0}}{C_{s}-C_{0}}=1-\operatorname{erf}\left(\frac{x}{2 \sqrt{D t}}\right)$ 


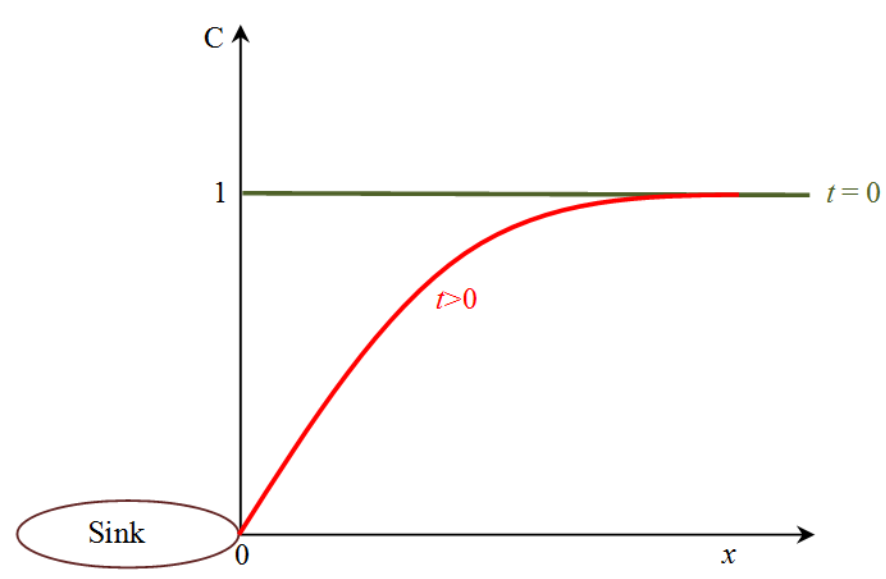

Fig. 11. The boundary condition of diffusion field of ions in bone. A sink represents a lacuna or bone marrow cavity

For $C(x, t)=0.7$, Equation 5-7 becomes:

$\operatorname{erf}\left(\frac{x}{2 \sqrt{D t}}\right)=0.7$

i.e.,

$\frac{x}{2 \sqrt{D t}} \approx 0.75$

A typical distance between lacunae and biomaterials particles was $\sim 50 \mu \mathrm{m}$ (Fig. 8). If $x=50 \mu \mathrm{m}$, then $t \approx 120 \mathrm{~s}$. Hence, once a biomaterial particle is ionised, it only takes several minutes for ions to migrate to a sink (lacuna or bone marrow) to reduce their concentration to the physiological level $(70-75 \mathrm{wt} \%)$. The slow mineralisation process after 40 days might be due to the rapid release of the $\mathrm{Ca}^{2+}$ and $\mathrm{PO}_{4}{ }^{3-}$ ions from Region II at 40 days post implantation. Considering both ions are readily soluble, an ideal $\mathrm{CaP}$ material should have a relatively slow degradation rate to provide a moresustained source of mineral ions for new bone tissue, rather than an initial pulse. This slow-release may enhance the mineralisation of new bone at later stages. Future experiments will explore the use of $\mathrm{CaP}$ of high crystallinity and other forms of $\mathrm{CaP}$, such as $\mathrm{OCP}$, which has been shown to be more stable and able to stimulate osteoblasts in a mouse calvaria model (Sasano et al., 1999).

\section{Conclusion}

This study describes a histological investigation of the remodelling process of critical-sized defects in rat calvarial bone at both the optical and electron microscopic levels, following repair with degradable biomaterials containing $\mathrm{CaP}$, with a focus on its influence on mineralisation. We found that implanted $\mathrm{CaP} /$ chitosan particles remarkably accelerated the bone growth rate of the defects at the initial stage, with an estimated growth rate of $0.8 \mathrm{mg} \mathrm{day}^{-1}$, which is almost ten-fold higher than the bone growth rate $\left(0.1 \mathrm{mg} \mathrm{day}^{-1}\right)$ in the non-implanted control group. However, we also noticed that implanted $\mathrm{CaP}$ did not enhance the mineralisation of regenerated cortical bone tissue at the later stage of bone remodelling. A theoretical analysis using Fick's law indicated that the excessive amounts of mineral ions due to the fast degradation of the biomaterial may be released rapidly from the new bone tissue. Hence, a $\mathrm{CaP}$ material with a reduced degradation rate could offer a sustained resource of mineral ions and thus enhance bone mineralisation at later stages.

\section{Author's Contributions}

TF, QZC and CHV designed the study. Samples were prepared by MAQ. Animal study experiments were carried out by TF. The cell culture experiments were carried out by YL and GAT. The data were analysed by TF, YL, GAT, MAO and QZC. The manuscript was written by QZC with contributions from TF, CHV, YL, GAT, DFN and MAO.

\section{Ethics}

There are no ethical issues.

\section{References}

Arinzeh, T.L., T. Tran, J. McAlary and G. Daculsi, 2005. A comparative study of biphasic calcium phosphate ceramics for human mesenchymal stem-cell-induced bone formation. Biomaterials, 26: 3631-3638.

DOI: $10.1016 /$ j.biomaterials.2004.09.035

Balaguer, T., F. Boukhechba, A. Clave, S. BouvetGerbettaz and C. Trojani et al., 2010. Biphasic calcium phosphate microparticles for bone formation: Benefits of combination with blood clot. Tissue Eng. Part A, 16: 3495-3505.

DOI: $10.1089 /$ ten.tea.2010.0227 
Barradas, A.M.C., H. Yuan, J. Van Der Stok, Q. Bach Le and H. Fernandes et al., 2012. The influence of genetic factors on the osteoinductive potential of calcium phosphate ceramics in mice. Biomaterials, 33: 5696-5705.

DOI: $10.1016 /$ j.biomaterials.2012.04.021

Bateman, J.P., F.F. Safadi, C. Susin and U.M. Wikesjo, 2012. Exploratory study on the effect of osteoactivin on bone formation in the rat criticalsize calvarial defect model. J. Periodontal. Res., 47: 243-247.

DOI: $10.1111 /$ j.1600-0765.2011.01428.x

Bohner, M., L. Galea and N. Doebelin, 2012. Calcium phosphate bone graft substitutes: Failures and hopes. J. Eur. Ceramic Society, 32: 2663-2671. DOI: 10.1016/j.jeurceramsoc.2012.02.028

Burg, K.J.L., S. Porter and J.F. Kellam, 2000. Biomaterial developments for bone tissue engineering. Biomaterials, 21: 2347-2359. DOI: 10.1016/S0142-9612(00)00102-2

Chai, Y.C., A. Carlier, J. Bolander, S.J. Roberts and L. Geris et al., 2012. Current views on calcium phosphate osteogenicity and the translation into effective bone regeneration strategies. Acta Biomaterialia, 8: 3876-3887.

DOI: $10.1016 /$ j.actbio.2012.07.002

Chen, Q.Z., C.H. Zhu and G.A. Thouas, 2012. Progress and challenges in biomaterials for tissue engineering. Progress Biomaterials.

DOI: $10.1186 / 2194-0517-1-2$

Develioglu, H., S. Unver Saraydin and U. Kartal, 2009. The bone-healing effect of a xenograft in a rat calvarial defect model. Dent Mater J., 28: 396-400. DOI: $10.4012 / \mathrm{dmj} .28 .396$

Egan, K.P., T.A. Brennan and R.J. Pignolo, 2012. Bone histomorphometry using free and commonly available software. Histopathology, 61: 1168-1173. DOI: $10.1111 / \mathrm{j} .1365-2559.2012 .04333 . \mathrm{x}$

Fernandez-Seara, M.A., S.L. Wehrli and F.W. Wehrli, 2002. Diffusion of exchangeable water in cortical bone studied by nuclear magnetic resonance. Biophys. J., 82: 522-529.

DOI: 10.1016/S0006-3495(02)75417-9

Fielding, G. and S. Bose, 2013. $\mathrm{SiO}_{2}$ and $\mathrm{ZnO}$ dopants in three-dimensionally printed tricalcium phosphate bone tissue engineering scaffolds enhance osteogenesis and angiogenesis in vivo. Acta Biomater., 9: 9137-9148. DOI: 10.1016/j.actbio.2013.07.009

Geffre, C.P., J. Ochoa, D.S. Margolis and J.A. Szivek, 2010. Evaluation of the osteogenic performance of calcium phosphate-chitosan bone fillers. J. Investigative Surgery, 23: 134-141.

DOI: $10.3109 / 08941930903564100$
Gosain, A.K., L. Song, P. Yu, B.J. Mehrara and C.Y. Maeda et al., 2000. Osteogenesis in cranial defects: Reassessment of the concept of critical size and the expression of TGF- $\beta$ isoforms. Plast Reconstr. Surg., 106: 360-371.

DOI: $10.1097 / 00006534-200008000-00018$

Habibovic, P. and K. de Groot, 2007. Osteoinductive biomaterials--properties and relevance in bone repair. J. Tissue Eng. Regen. Med., 1: 25-32. DOI: $10.1002 /$ term.5

Hansson, L.I., K. Menander-Sellman, A. Stenstrom and K.G. Thorngren, 1972. Rate of normal longitudinal bone growth in the rat. Calcif. Tissue Res., 10: 238-2351. DOI: 10.1007/BF02012553

Ignjatovic, N.L., Z.R. Ajdukovic, V.P. Savic and D.P. Uskokovic, 2010. Size effect of calcium phosphate coated with poly-DL-lactide-co-glycolide on healing processes in bone reconstruction. J. Biomed Mater Res. B Applied Biomater, 94: 108-117.

PMID: 20524184

Jones, L., J.S. Thomsen, L. Mosekilde, C. Bosch and B. Melsen, 2007. Biomechanical evaluation of rat skull defects, 1, 3 and 6 months after implantation with osteopromotive substances. J. Craniomaxillofac. Surg., 35: 350-357.

DOI: $10.1016 /$ j.jcms.2007.06.004

Kim, I.Y., S.J. Seo, H.S. Moon, M.K. Yoo and I.Y. Park et al., 2008. Chitosan and its derivatives for tissue engineering applications. Biotechnol. Adv., 26: 121. DOI: 10.1016/j.biotechadv.2007.07.009

Kochi, G., S. Sato, T. Fukuyama, C. Morita and K. Honda et al., 2009. Analysis on the guided bone augmentation in the rat calvarium using a microfocus computerized tomography analysis. Oral Surg. Oral Med. Oral Pathol. Oral Radiol., 107: e42-48. DOI: 10.1016/j.tripleo.2009.02.010

Lange, T., A.F. Schilling, F. Peters, J. Mujas and D. Wicklein et al., 2011. Size dependent induction of proinflammatory cytokines and cytotoxicity of particulate beta-tricalciumphosphate in vitro. Biomaterials, 32: 4067-4075.

DOI: $10.1016 /$ j.biomaterials.2011.02.039

Liang, S.L., W.D. Cook, G.A. Thouas and Q.Z. Chen, 2010. The mechanical characteristics and in vitro biocompatibility of poly(glycerol sebacate)Bioglass ${ }^{\circledR}$ elastomeric composites. Biomaterials, 31: 8516-8529.

DOI: $10.1016 /$ j.biomaterials.2010.07.105

Lu, J., M. Descamps, J. Dejou, G Koubi and P. Hardouin et al., 2002. The biodegradation mechanism of calcium phosphate biomaterials in bone. J. Biomed Mater Res., 63: 408-412. DOI: 10.1002/jbm.10259 
Mikhailenko, N.Y., E.E. Stroganova and N.V. Buchilin, 2013. Solubility of calcium phosphate glasses and glass ceramic materials in water and physiological media. Glass Ceramics, 70: 158-163.

DOI: $10.1007 / \mathrm{s} 10717-013-9531-8$

Mills, L.A. and A.H. Simpson, 2012. In vivo models of bone repair. J. Bone Joint Surg. 94: 865-874. DOI: 10.1302/0301-620X.94B7.27370

Pannarale, L., S. Morini, E. D'Ubaldo, E. Gaudio and G. Marinozzi, 1997. SEM corrosion-casts study of the microcirculation of the flat bones in the rat. Anat. Rec., 247: 462-471. DOI: 10.1002/(SICI)10970185(199704)247:4<462::AID-AR4>3.0.CO;2-T

Raman, A., 1969. Appositional growth rate in rat bones using the tetracycline labelling method. Acta Orthop Scand, 40: 193-197. DOI: $10.3109 / 17453676908989498$

Sasano, Y., S. Kamakura, H. Homma, O. Suzuki and I. Mizoguchi et al., 1999. Implanted Octacalcium Phosphate (OCP) stimulates osteogenesis by osteoblastic cells and/or committed osteoprogenitors in rat calvarial periosteum. Anatomical Record, 256: $1-6$ DOI: $10.1002 /($ SICI) $1097-$ 0185(19990901)256:1<1::AID-AR1>3.0.CO;2-X
Thormann, U., S. Ray, U. Sommer, T. Elkhassawna and T. Rehling et al., 2013. Bone formation induced by strontium modified calcium phosphate cement in critical-size metaphyseal fracture defects in ovariectomized rats. Biomaterials, 34: 8589-8598. DOI: $10.1016 /$ j.biomaterials.2013.07.036

Vajgel, A., N. Mardas, B.C. Farias, A. Petrie and R. Cimoes et al., 2013. A systematic review on the critical size defect model. Clin. Oral. Implants Res., 25: 879-893. PMID: 23742162

Vignoletti, F., P. Matesanz, D. Rodrigo, E. Figuero and C. Martin et al., 2012. Surgical protocols for ridge preservation after tooth extraction. A systematic review. Clin. Oral. Implants Res., 5: 22-38.

DOI: $10.1111 / \mathrm{j} .1600-0501.2011 .02331 . \mathrm{x}$

Yuan, H., C.A. van Blitterswijk, K. de Groot and J.D. de Bruijn, 2006. A comparison of bone formation in Biphasic Calcium Phosphate (BCP) and Hydroxyapatite (HA) implanted in muscle and bone of dogs at different time periods. J. Biomed. Materials Res. Part A, 78A: 139-147.

DOI: 10.1002/jbm.a.30707 